\title{
The luminous late-time emission of the type-Ic supernova iPTF15dtg - evidence for powering from a magnetar?
}

\author{
F. Taddia ${ }^{1}$, J. Sollerman ${ }^{1}$, C. Fremling ${ }^{2,3}$, E. Karamehmetoglu ${ }^{1}$, C. Barbarino ${ }^{1}$, R. Lunnan ${ }^{1}$, \\ S. West ${ }^{1}$, and A. Gal-Yam ${ }^{4}$ \\ 1 The Oskar Klein Centre, Department of Astronomy, Stockholm University, AlbaNova 10691, Stockholm, Sweden \\ e-mail: francesco.taddia@astro.su.se \\ 2 Division of Physics, Math and Astronomy, California Institute of Technology, 1200 East California Boulevard, Pasadena, \\ 3 Cahill Center for Astrophysics, California Institute of Technology, Pasadena, CA 91125, USA \\ ${ }^{4}$ Benoziyo Center for Astrophysics, Weizmann Institute of Science, Rehovot 76100, Israel
} CA 91125, USA

Received 21 June 2018 / Accepted 4 November 2018

\begin{abstract}
Context. The transient iPTF15dtg is a type-Ic supernova (SN) showing a broad light curve around maximum light, consistent with massive ejecta if we assume a radioactive-powering scenario.

Aims. We aim to study the late-time light curve of iPTF15dtg, which turned out to be extraordinarily luminous for a stripped-envelope (SE) SN, and investigate possible powering mechanisms.

Methods. We compare the observed light curves to those of other SE SNe and also to models for the ${ }^{56}$ Co decay. We analyze and compare the spectra to nebular spectra of other SE SNe. We build a bolometric light curve and fit it with different models, including powering by radioactivity, magnetar powering, and a combination of the two.

Results. Between 150 and $750 \mathrm{~d}$ post-explosion, the luminosity of iPTF15dtg declined by merely two magnitudes instead of the six magnitudes expected from ${ }^{56} \mathrm{Co}$ decay. This is the first spectroscopically regular SE SN found to show this behavior. The model with both radioactivity and magnetar powering provides the best fit to the light curve and appears to be the most realistic powering mechanism. An alternative mechanism might be circumstellar-medium (CSM) interaction. However, the spectra of iPTF15dtg are very similar to those of other SE SNe, and do not show signs of strong CSM interaction.

Conclusions. The object iPTF15dtg is the first spectroscopically regular SE SN whose light curve displays such clear signs of a magnetar contributing to its late-time powering. Given this result, the mass of the ejecta needs to be revised to a lower value, and therefore the progenitor mass could be significantly lower than the previously estimated $>35 M_{\odot}$.
\end{abstract}

Key words. supernovae: individual: SN 2011bm - supernovae: individual: iPTF15dtg - supernovae: individual: SN 2010mb supernovae: general

\section{Introduction}

Core-collapse (CC) stripped-envelope (SE) supernovae (SNe), such as Type IIb, Ib and Ic, are believed to be powered by radioactive decay of ${ }^{56} \mathrm{Ni}$ and its decay product ${ }^{56} \mathrm{Co}$. When radioactive elements such as ${ }^{56} \mathrm{Co}$ are the source of the SN emission, their characteristic decay times should govern the evolution of the SN luminosity, especially at late epochs when the ejecta are mostly transparent. In fact, the late-time observations of SE SNe have so far revealed that the luminosity is declining at the rate of ${ }^{56} \mathrm{Co}$ decay or in most cases faster than that, due to increasing gamma-ray leakage from the ejecta (e.g., Wheeler et al. 2015). Hitherto we have not observed a spectroscopically regular SE SN whose luminosity declines slower than the ${ }^{56} \mathrm{Co}$ decay $\left(0.0098 \mathrm{mag} \mathrm{day}^{-1}\right)$, which agrees well with the overall idea that CC SE SNe are radioactively powered.

The late-time luminosity of a CC SN could decline more slowly than the ${ }^{56} \mathrm{Co}$ decay rate if a different or additional powering source is at play. This could for example be circumstellar interaction or the energy input from a magnetar. Some typeIIn SNe exhibit long-lasting emission at high luminosity, because their ejecta interact with their surrounding medium and convert the kinetic energy into radiation (see e.g., Stritzinger et al.
2012, for the case of SNe 2005ip and 2006jd). Such interaction also produces features in the spectra (narrow emission lines) that are easy to identify. An example of a CSM-interacting SE SN is SN 2010mb (Ben-Ami et al. 2014), whose spectrum shows a remarkable blue color and whose light curve exhibits a slow decline. Superluminous SNe (SLSNe) are believed to be magnetar-powered, as their late phases are also particularly bright and difficult to reconcile with ${ }^{56}$ Co powering (Inserra et al. 2013).

(Taddia et al. 2016; hereafter T16) presented and analyzed the first 150 days of SN iPTF15dtg. This type-Ic SN exhibits a particularly broad light curve, similar to that of SN $2011 \mathrm{bm}$ (Valenti et al. 2012). Its broadness suggests massive ejecta in a scenario where radioactive decay powers the $\mathrm{SN}$. The very early epochs of iPTF15dtg also revealed a first peak on the timescale of a few days, possibly powered by the post-shock breakout cooling tail from an extended envelope. Signs of such early emission were also found in the early data of SN $2011 \mathrm{bm}$ (T16). The early spectra of iPTF15dtg are similar to those of normal type-Ic $\mathrm{SNe}$, and show no clear signs of interaction with circumstellar medium (CSM).

After about five months of follow up, iPTF15dtg went behind the Sun. This is when the initial monitoring was terminated and the early results were published in T16. After approximately 300 days 
post-explosion, we recovered the $\mathrm{SN}$ with the P48 telescope at Palomar - to our great surprise. Where we had expected the SN to decline at least as fast as the decay time of ${ }^{56} \mathrm{Co}$, it appeared much brighter. We therefore started our late-time monitoring and kept following it until $\sim 840$ days, as it declined very slowly. iPTF15dtg is indeed declining slower than radioactive decay, revealing a new powering mechanism for its late epochs. We argue below that this mechanism could be the spin-down of a magnetar, combined with the classic radioactive emission.

In this paper, we present the late-time data acquisition and reduction for iPTF15dtg in Sect. 2. We present and analyze its late-time light curves and the spectra in Sects. 3 and 4, respectively. We build (Sect. 5) and model the bolometric light curve in Sect. 6. We then discuss the powering mechanism and the implications for the progenitor star in Sect. 7 and finally give our conclusions in Sect. 8.

\section{Data acquisition and reduction}

Our late-time data for iPTF15dtg are from a number of facilities. Photometry in $g$ and $r$ band was obtained with the P48 telescope equipped with the 96 Mpixel mosaic camera CFH12K (Rahmer et al. 2008). The Nordic Optical Telescope (NOT) with ALFOSC (Andalucia Faint Object Spectrograph and Camera), the Telescopio Nazionale Galileo (TNG) with DOLORES (Device Optimized for the LOw RESolution), and the Palomar 200-inch Hale Telescope (P200) provided further gri band photometry. A single epoch of $r$-band Photometry Was Obtained with the Palomar 60" telescope (P60; Cenko et al. 2006) equipped with GRBcam. P48 photometry was reduced with the Palomar Transient Factory Image Differencing and Extraction (PTFIDE) pipeline which performs template subtraction and PSF photometry (Masci et al. 2017). The images from the NOT, TNG, P200, and P60 were reduced with the FPipe pipeline (Fremling et al. 2016).

Standard stars from the PANSTARRS catalog ${ }^{1}$ were used as reference stars to calibrate the photometry.

From the PANSTARRS archive we also obtained the deep images used to template-subtract our SN frames. The final light curves are presented after combining the magnitudes obtained from the same night. A log of the photometry is provided in Table 1.

Late-time spectra are from Keck I equipped with LRIS (Low Resolution Imaging Spectrometer, Oke et al. 1995) and Keck II equipped with DEIMOS, as well as from the TNG equipped with DOLORES (grism LR-B and LR-R, slit 1."0) and NOT equipped with ALFOSC (grism \#4, slit 1."0). All the spectra were reduced in a standard manner with dedicated pipelines. The reduction includes bias correction and flat-fielding, one-dimensional (1D) spectral extraction, wavelength calibration through a comparison with an arc-lamp, and flux calibration through a sensitivity function derived from spectrophotometric standard stars. Absolute flux calibration was obtained by computing synthetic photometry in the $r$ band from the spectra, by comparing the obtained photometry to the $r$-band light curves and, when needed, by multiplying the spectra for the flux ratio correspondent to the difference in magnitude. A log of the spectroscopy is provided in Table 2.

\section{Late-time light curves}

The early-time $(<150 \mathrm{~d})$ light curves of iPTF15dtg were presented in T16. Later, P48 epochs in $g$ and $r$ bands cover

\footnotetext{
1 http://archive.stsci.edu/panstarrs/search.php
}

300-400 days since explosion. A single $i$-band detection from the NOT was obtained in that same period. An epoch of gri band photometry from the NOT was also obtained around $500 \mathrm{~d}$, together with a single P60 $r$-band point. TNG, NOT, and P200 gri data cover the epochs between 650 and $840 \mathrm{~d}$. In Fig. 1 we plot the absolute-magnitude light curves obtained using the apparent magnitudes given in Table 1, together with the explosion epoch, the distance modulus, and the extinction from T16.

As mentioned above, the late time $(>300 \mathrm{~d})$ photometry of iPTF15dtg was surprisingly bright. A SE SN is expected to decline at least as fast as the ${ }^{56}$ Co decay (see e.g., Wheeler et al. 2015), which in Fig. 1 is marked by dashed lines for the $g$ (green) and $r$ (red) bands. Assuming that the SN had settled on the linear decay just before disappearing behind the Sun at $150 \mathrm{~d}$, we recovered it around 300-400 d, 1.4 mag brighter than expected in the $r$ band.

The brightness of iPTF15dtg continued to decline extremely slowly between 500 and $650 \mathrm{~d}$. A relatively short $(3 \times 600 \mathrm{~s})$ set of gri images of iPTF15dtg taken with the NOT at $687 \mathrm{~d}$, which is shown in Fig. 2, still show a bright SN as compared to its host galaxy. This is unique for SE SNe, which are typically extremely hard to follow at such late epochs unless they are very nearby (iPTF15dtg exploded at $232 \mathrm{Mpc}, \mathrm{T} 16$ ). The decline in the $r$ band between 150 and 750 days is only $\sim 2$ mag, instead of the 6 mag expected from the ${ }^{56}$ Co decay.

The anomalous behavior of the light curves of iPTF15dtg at late times is even more evident when compared to the late-time light curves of other SE SNe, as shown in Fig. 3. The SN that most resembles iPTF15dtg at early epochs, that is, SN 2011bm, has a similar broad light curve peak but declines at the ${ }^{56} \mathrm{Co}$ decay rate. This is as expected from a $\mathrm{SN}$ with a massive envelope which will trap all the gamma-rays. Other SE SNe with narrower peaks decline even faster, as their ejecta are unable to trap all the gamma rays. The SN iPTF15dtg is instead well above the late-time luminosity expected if the same amount of ${ }^{56} \mathrm{Ni}$ (and its decay product ${ }^{56} \mathrm{Co}$ ) were to power both the peak and the late emission. The decay luminosity from the amount of radioactive material necessary to power its peak, as derived in T16, is marked by a dashed black line in Fig. 3. The only other SE SN that is above the ${ }^{56}$ Co decay luminosity in this figure is SN $2010 \mathrm{mb}$. However, that SN also had a very peculiar early light curve. It had a more than 100 days long rise, a sort of plateau around peak lasting another 100 days, and signs of strong CSM interaction in the spectrum (see Sect. 4). In particular, SN 2010mb showed a very blue color of the spectrum, seen also in the late time light curves (Ben-Ami et al. 2014, see also Fig. 4). Therefore SN 2010mb cannot be considered a regular SE SN. Another SE SN declining slower than ${ }^{56}$ Co decay at late epochs is SN 2014C, whose $R$ band dropped by 2.16 mag between 139 and 509 d (Margutti et al. 2017, iPTF15dtg dropped by $1.29 \mathrm{mag}$ in the same time range). However, the late-time emission of SN 2014C is characterized by strong CSM interaction revealed by a prominent $\mathrm{H} \alpha$ emission in the spectrum (Milisavljevic et al. 2015).

In Fig. 4 we compare the colors of iPTF15dtg to those of type-Ic SNe 2011bm and 2010mb. The late-time colors of SN $2011 \mathrm{bm}$ are only slightly redder than those of iPTF15dtg. The SN 2010mb shows a much bluer $g-r$ color than iPTF15dtg at $270 \mathrm{~d}$.

\section{Late-time spectra}

Whereas the late-time light curves are unique, the late-time spectra of iPTF15dtg are very similar to those observed in regular 
Table 1. Late-time optical photometry of iPTF15dtg.

\begin{tabular}{|c|c|c|c|c|c|c|c|c|}
\hline $\begin{array}{c}\text { JD } \\
\text { (days) }\end{array}$ & $\begin{array}{c}g \\
(\mathrm{mag})\end{array}$ & $\begin{array}{c}\operatorname{err}_{g} \\
(\mathrm{mag})\end{array}$ & $\begin{array}{c}\text { JD } \\
\text { (days) }\end{array}$ & $\begin{array}{c}r \\
\text { (mag) }\end{array}$ & $\begin{array}{c}\operatorname{err}_{r} \\
(\mathrm{mag})\end{array}$ & $\begin{array}{c}\text { JD } \\
\text { (days) }\end{array}$ & $\begin{array}{c}i \\
(\mathrm{mag})\end{array}$ & $\begin{array}{c}\operatorname{err}_{i} \\
(\mathrm{mag})\end{array}$ \\
\hline \multicolumn{9}{|c|}{ P48 } \\
\hline 2457642.97 & 20.95 & 0.24 & 2457627.00 & 20.07 & 0.18 & & & \\
\hline 2457656.99 & 20.94 & 0.30 & 2457629.99 & 20.35 & 0.12 & & & \\
\hline 2457662.99 & 20.72 & 0.16 & 2457632.98 & 20.40 & 0.17 & & & \\
\hline 2457665.98 & 20.74 & 0.07 & 2457635.99 & 20.37 & 0.16 & & & \\
\hline 2457668.97 & 20.70 & 0.29 & 2457642.02 & 20.31 & 0.23 & & & \\
\hline 2457672.03 & 20.44 & 0.29 & 2457643.01 & 20.19 & 0.11 & & & \\
\hline 2457674.98 & 20.73 & 0.21 & 2457663.02 & 20.35 & 0.17 & & & \\
\hline 2457687.90 & 21.23 & 0.34 & 2457669.02 & 20.39 & 0.26 & & & \\
\hline 2457693.89 & 20.78 & 0.18 & 2457671.99 & 20.26 & 0.13 & & & \\
\hline 2457694.91 & 21.20 & 0.36 & 2457674.96 & 20.54 & 0.15 & & & \\
\hline 2457695.90 & 20.80 & 0.29 & 2457681.95 & 20.76 & 0.35 & & & \\
\hline 2457696.88 & 21.01 & 0.20 & 2457682.95 & 20.37 & 0.28 & & & \\
\hline 2457697.91 & 20.89 & 0.19 & 2457683.92 & 20.57 & 0.20 & & & \\
\hline 2457698.88 & 20.81 & 0.16 & 2457684.95 & 20.81 & 0.33 & & & \\
\hline 2457699.87 & 21.04 & 0.29 & 2457687.94 & 20.93 & 0.26 & & & \\
\hline \multirow[t]{10}{*}{2457700.90} & 21.03 & 0.30 & 2457694.95 & 20.59 & 0.27 & & & \\
\hline & & & 2457695.94 & 20.58 & 0.26 & & & \\
\hline & & & 2457696.92 & 20.68 & 0.29 & & & \\
\hline & & & 2457697.95 & 20.63 & 0.23 & & & \\
\hline & & & 2457698.93 & 20.87 & 0.26 & & & \\
\hline & & & 2457699.91 & 20.30 & 0.21 & & & \\
\hline & & & 2457700.94 & 20.75 & 0.36 & & & \\
\hline & & & 2457701.91 & 20.69 & 0.33 & & & \\
\hline & & & 2457715.74 & 20.72 & 0.20 & & & \\
\hline & & & 2457718.73 & 20.88 & 0.28 & & & \\
\hline \multicolumn{9}{|c|}{ P60 } \\
\hline & & & 2457819.69 & 20.66 & 0.19 & & & \\
\hline \multicolumn{9}{|c|}{ NOT } \\
\hline 2457694.55 & 21.26 & 0.08 & 2457694.55 & 20.58 & 0.04 & 2457694.55 & 20.73 & 0.05 \\
\hline 2457822.37 & 21.42 & 0.20 & 2457822.38 & 20.98 & 0.13 & 2457822.38 & 21.22 & 0.16 \\
\hline 2457985.58 & 22.02 & 0.19 & 2457985.59 & 21.60 & 0.07 & 2457985.59 & 21.97 & 0.11 \\
\hline 2458044.54 & 21.93 & 0.17 & 2458044.55 & 21.72 & 0.13 & 2458044.56 & 22.12 & 0.23 \\
\hline \multirow[t]{3}{*}{2458056.71} & 22.02 & 0.16 & 2458056.72 & 21.70 & 0.12 & 2458056.73 & 22.33 & 0.21 \\
\hline & & & 2458067.64 & 22.12 & 0.23 & & & \\
\hline & & & 2458093.42 & 22.08 & 0.20 & & & \\
\hline \multicolumn{9}{|c|}{ TNG } \\
\hline 2458012.63 & 21.54 & 0.07 & 2458012.65 & 21.31 & 0.07 & 2458012.64 & 21.48 & 0.07 \\
\hline 2458023.56 & 21.54 & 0.08 & 2458023.57 & 21.83 & 0.13 & 2458023.58 & 21.25 & 0.05 \\
\hline 2458077.55 & 22.26 & 0.18 & 2458077.57 & 21.99 & 0.10 & 2458115.43 & 22.56 & 0.19 \\
\hline 2458115.42 & 22.51 & 0.21 & 2458115.44 & 22.35 & 0.15 & 2458171.34 & 22.87 & 0.21 \\
\hline 2458171.35 & 22.84 & 0.18 & 2458171.36 & 22.48 & 0.18 & & & \\
\hline \multicolumn{9}{|c|}{$\mathrm{P} 200$} \\
\hline 2458052.84 & 21.83 & 0.29 & 2458052.85 & 21.57 & 0.15 & 2458052.85 & 22.20 & 0.13 \\
\hline & & & 2458052.85 & 21.56 & 0.10 & & & \\
\hline
\end{tabular}

SE SNe (e.g., Taubenberger et al. 2009; Jerkstrand et al. 2015). High-quality spectra from Keck I (LRIS) and Keck II (DEIMOS) were obtained at 360, 650, and $737 \mathrm{~d}$ after explosion. Additional spectra from NOT (ALFOSC) and TNG (DOLORES) were obtained between these epochs. All the spectra will be released via WISeREP ${ }^{2}$ (Yaron \& Gal-Yam 2012). We show in Fig. 5 the absolute-flux calibrated spectra in the rest-frame. In the figure we also identify the main emission lines. The [O I] $\lambda 6300$ line is the most prominent feature, together with the

\footnotetext{
2 https://wiserep.weizmann.ac.il
}

[Ca II] $\lambda \lambda 7291,7323$ and the Ca II triplet. The oxygen lines from [OI] $\lambda 5577, \mathrm{O}_{\mathrm{I}} \lambda 7774$ and $\mathrm{O}_{\mathrm{I}} \lambda 9263$ are also present. In the bluer part of the spectra we observe the presence of $\left.\mathrm{Mg}_{\mathrm{I}}\right] \lambda 4571$ and Fe II lines. Narrow emission lines from the host galaxy are present in some of the spectra (already observed at early epochs, T16), when the slit was oriented in a way that includes a nearby $\mathrm{H}$ II region. These lines are certainly from the host and not due to CSM interaction, as we observe [O II] $\lambda 3727$, which is characteristic of the spectra from $\mathrm{H}$ in regions. We removed the hostgalaxy narrow lines from the SN spectra, except from the third spectrum where they are labeled in red. 


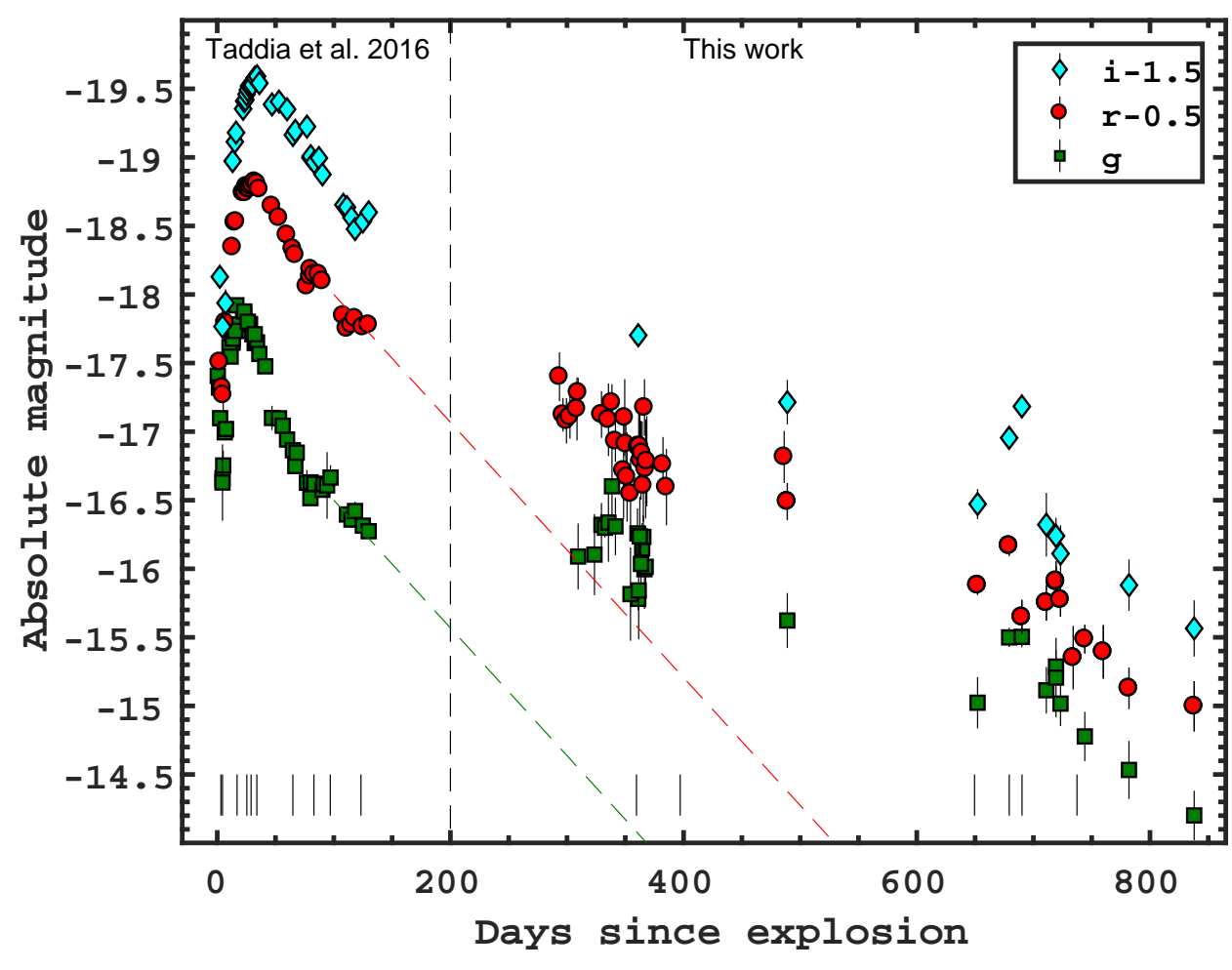

Fig. 1. Absolute-magnitude light curves of iPTF15dtg in the gri filters in the observer frame. Each light curve was shifted by the amount indicated in the legend for clarity. The spectral epochs are reported as vertical black segments at the bottom. The ${ }^{56}$ Co decay rate is shown as dashed lines and scaled to both early $g$ and $r$ band photometry.

Table 2. Late-time optical spectroscopy of iPTF15dtg.

\begin{tabular}{cccccc}
\hline \hline Date (UT) & $\begin{array}{c}\text { JD-2 457 000 } \\
\text { (days) }\end{array}$ & $\begin{array}{c}\text { Phase }^{a} \\
\text { (days) }\end{array}$ & Telescope & Instrument & $\begin{array}{c}\text { Range } \\
(\AA)\end{array}$ \\
\hline 31 Oct. 2016 & 693.02 & +359.6 & Keck1 & LRIS & $3080-10302$ \\
07 Dec. 2016 & 730.53 & +397.1 & NOT & ALFOSC & $3801-9711$ \\
17 Aug. 2017 & 982.97 & +649.5 & Keck1 & LRIS & $3071-10268$ \\
16 Sep. 2017 & 1012.74 & +679.3 & TNG & DOLORES & $3801-9719$ \\
26 Sep. 2017 & 1023.71 & +690.3 & TNG & DOLORES & $3501-8341$ \\
13 Nov. 2017 & 1070.83 & +737.4 & Keck2 & DEIMOS & $4403-9905$ \\
\hline
\end{tabular}

Notes. ${ }^{(a)}$ Days from explosion date in the observer frame.

The [OI] $\lambda 6300$ line shows a double peak after $650 \mathrm{~d}$. We were able to fit the $\left[\mathrm{O}_{\mathrm{I}}\right] \lambda 6300,6364$ feature (see Fig. 6) with two Gaussian components for each line, fixing the ratio between the $6364 \AA$ and the $6300 \AA$ component to $1: 3$ (suitable for the optically thin limit) and keeping their central wavelengths at constant distance as well as keeping their respective FWHM equal. The continuum is subtracted by fitting a first-order polynomial. The physical origin of these two Gaussian components might be explained by two lobes of oxygen-rich material belonging to the ejecta, one moving in the direction of the observer while the other one moves in the opposite direction. This would result in a blueshifted and a redshifted component.

Additionally, the [O I] $\lambda 5577$ line is strong in the first spectrum and fades until it almost disappears in the following spectra. Since this line is more prominent at high densities, its disappearance supports the assumption of optically thin emission used to fit the $[\mathrm{O}$ I] $\lambda 6300,6364$ feature in the very late spectra (see Fig. 6).

The MgI] $\lambda 4571$ line shows a boxy profile in the first spectrum, which later disappears. The OI $\lambda 7774$ line becomes narrower with time, similar to the various $\mathrm{Ca}$ II lines in the spectra.
When we compare the spectrum of iPTF15dtg with that of SN $2011 \mathrm{bm}$ around $300 \mathrm{~d}$ in Fig. 7, the two spectra are indeed very similar. Among the small differences, the [O I] $\lambda 5577$ line is stronger (as compared to the continuum) in iPTF15dtg, and its Mg I] $\lambda 4571$ line has a boxy profile. Around $4000-5500 \AA$ our SN is marginally bluer than SN $2011 \mathrm{bm}$. Furthermore, the [O I] $\lambda 6300$ line is relatively (compared to the continuum) stronger in SN $2011 \mathrm{bm}$. In Fig. 7 we also compare the spectrum of iPTF15dtg at $360 \mathrm{~d}$ with late-time spectra of SN Ic 2004aw (Taubenberger et al. 2006) and SN Ic-BL 1998bw (Patat et al. 2001). These spectra are also similar to that of iPTF15dtg, which is only slightly bluer. We also compare the spectrum of iPTF15dtg with that of SN 2014C from Milisavljevic et al. (2015), in order to show the strong $\mathrm{H} \alpha$ emission of that $\mathrm{SN}$, indicative of CSM interaction. Supernova 2014C does not have a spectrum as blue as that of SN 2010mb. We also notice that the late-time O I 7774 emission of iPTF15dtg resembles that of SLSN-I 2015bn (Nicholl et al. 2016a), which is suggested to be powered by a central engine such as a magnetar. The late-time spectra of iPTF15dtg also resemble those of $\mathrm{SN} \mathrm{Ib}$ 2012au at similar phases (Milisavljevic et al. 2018, their Fig. 3). Interestingly, the 


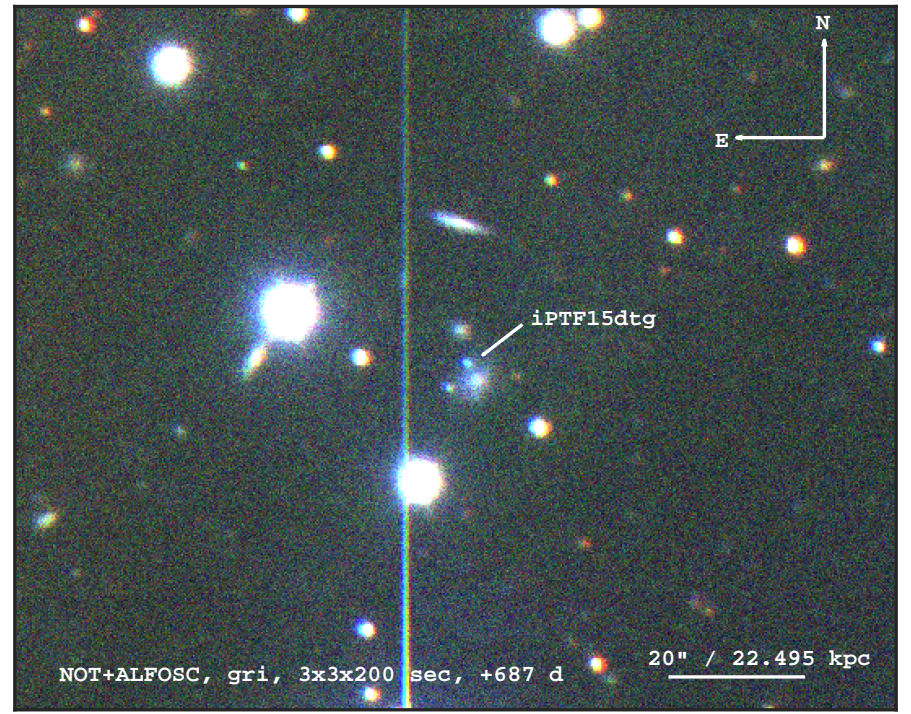

Fig. 2. iPTF15dtg in a color-combined image from the NOT equipped with ALFOSC taken $687 \mathrm{~d}$ post-explosion. The $\mathrm{SN}$ is still remarkably bright compared to its host. We report the scale and the orientation of the image in the right-hand corners. A saturated star outside of the fieldof-view produced the vertical feature in the middle of the frame.

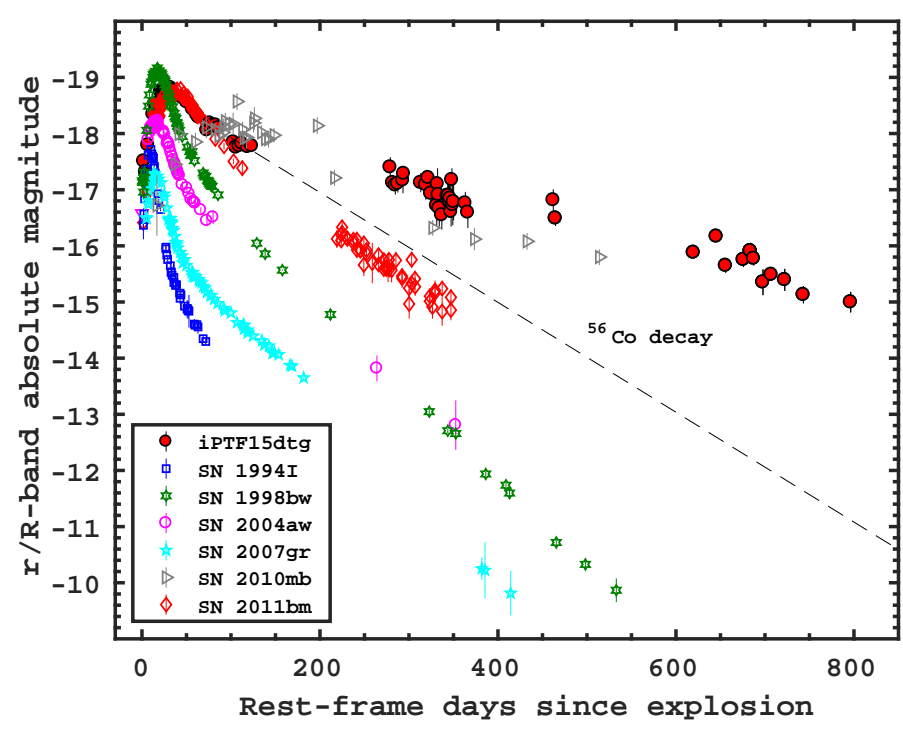

Fig. 3. The $r / R$-band absolute-magnitude light curves of a few typeIc SNe from the literature, compared to that of iPTF15dtg, which is declining much more slowly. The references for these light curves are listed in Fig. 3 of T16. Supernova 2010mb data are from Ben-Ami et al. (2014). The late-time ${ }^{56} \mathrm{Ni}+{ }^{56} \mathrm{Co}$ luminosity expected from the amount of ${ }^{56} \mathrm{Ni}$ estimated from the peak of iPTF15dtg is marked by a dashed black line.

recent spectra of SN 2012au at six years after discovery reveal strong forbidden oxygen emission consistent with a pulsar wind nebula (Milisavljevic et al. 2018).

The spectra of iPTF15dtg do not reveal any clear sign of strong CSM interaction. Such an interaction could have explained the large excess in luminosity described in Sect. 3. The few SE SNe that are believed to be powered by CSM interaction, such as type Ibn or the peculiar type-Ic SN 2010mb (Ben-Ami et al. 2014), do show either strong (narrow) He lines in emission or a conspicuous blue continuum on top of the nebular spectrum. We show the spectrum of SN 2010mb at

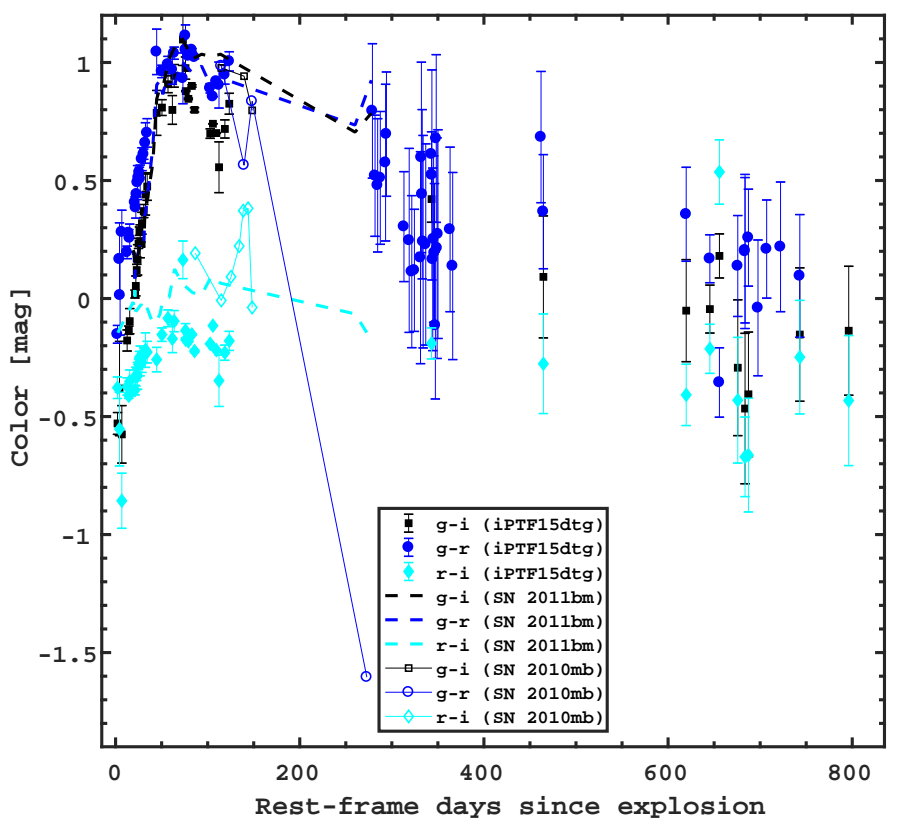

Fig. 4. Color comparison between iPTF15dtg, SN 2011bm (Valenti et al. 2012), and SN 2010mb (Ben-Ami et al. 2014). At late epochs ( 270 d) SN $2010 \mathrm{mb}$ exhibits a much bluer $g-r$ color than the other two SNe.

$+300 / 400 \mathrm{~d}$ in Fig. 7 (in red), where the continuum displays a blue component, which was interpreted as a sign of CSM interaction in Ben-Ami et al. (2014). This is neither present in iPTF15dtg nor in SN 2011 bm (or SN 1998bw and SN 2004aw). T16 reported the spectra of iPTF15dtg as being similar to those of SN $2011 \mathrm{bm}$ also at epochs earlier than $150 \mathrm{~d}$. In short, in terms of spectroscopy iPTF15dtg simply resembles a spectroscopically regular SE SN.

\section{Late-time bolometric light curve}

To address the nature of the powering mechanism of iPTF15dtg at late epochs, we construct a bolometric light curve. The early bolometric light curve is adopted from T16, where it was computed using $g-r$ colors and the bolometric corrections by Lyman et al. (2014). At later epochs, where the method of Lyman et al. (2014) does not apply, we estimate (pseudo)bolometric corrections using the late-time SN spectra. First we calibrate the two Keck I spectra using the $r$-band photometry. We then integrate the entire flux between 3000 and $9800 \AA$ in the spectra. This flux, transformed into magnitudes and subtracted by the $r$-band magnitude from the photometry at the same epoch, gives a (pseudo) bolometric correction that can be applied to the $r$ band to compute a (quasi) bolometric light curve. We use the spectrum at $360 \mathrm{~d}$ to compute the pseudo-bolometric correction between 300 and $500 \mathrm{~d}$, and the spectrum obtained at $650 \mathrm{~d}$ to compute the pseudo-bolometric correction at later epochs. We then apply these corrections to the $r$-band light curve to obtain the bolometric light curve plotted in Fig. 8. We note that with our method our pseudo-bolometric light curve at late epochs does not include the ultraviolet and near-infrared flux, and can therefore be regarded as a lower limit of the actual bolometric luminosity. Despite this fact, it is still remarkably brighter than the expected ${ }^{56}$ Co luminosity at late epochs (see Sect. 6). 


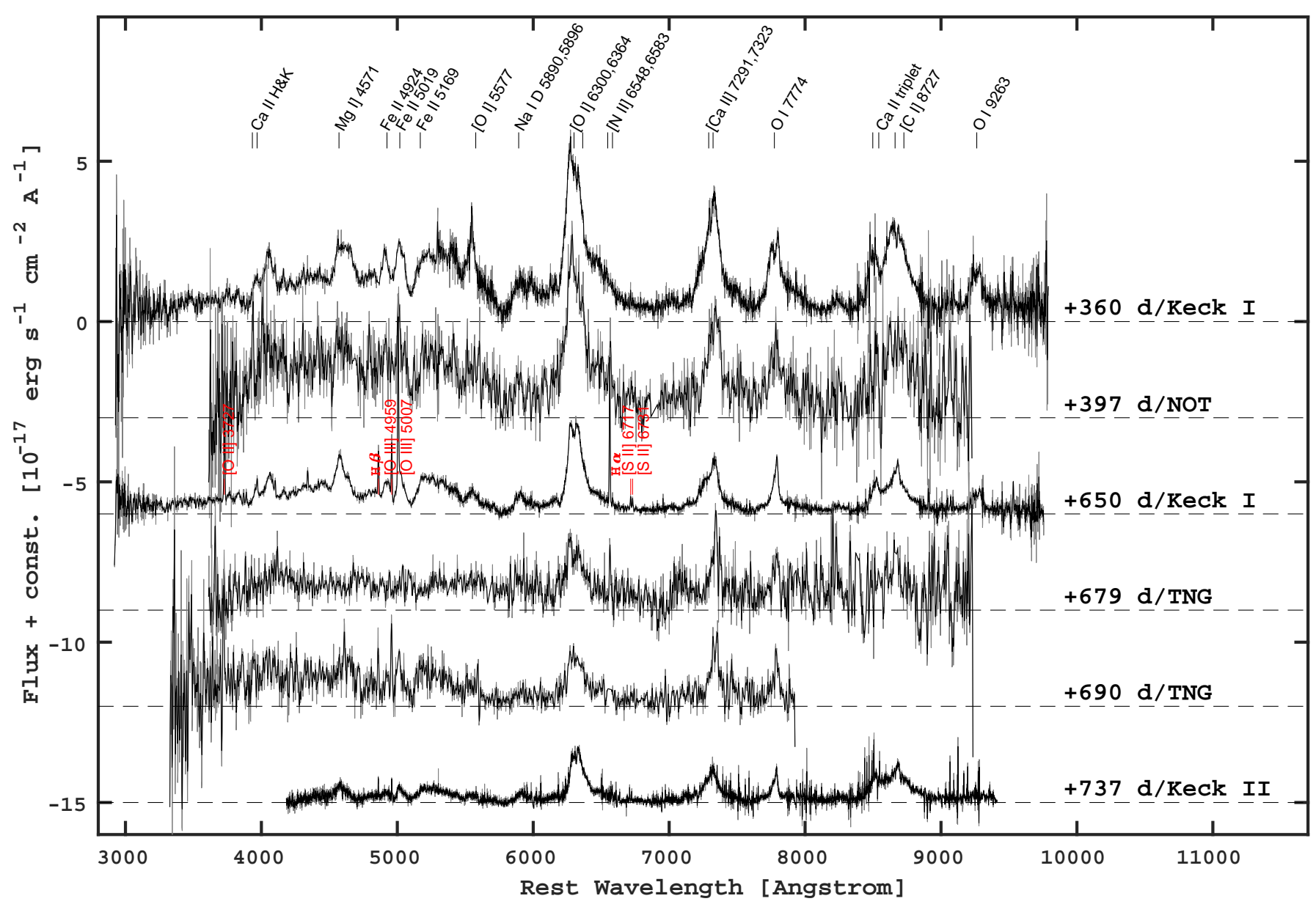

Fig. 5. Late-time spectral sequence of iPTF15dtg. All the spectra are absolute-flux calibrated and shown in the rest frame. The zero-flux level for each spectrum is marked by a horizontal dashed line, as well as its phase and telescope. The main SN features are labeled in black at the top, those related to the host-galaxy emission, well visible in the third spectrum, are labeled in red.

\section{Modeling}

We attempt to model the bolometric light curve with three different models. The three models are shown in Fig. 8.

First we confirm that radioactive decay alone cannot explain the late-time light curve of iPTF15dtg. Even though it provides a good fit to the early light curve (as also shown in T16), it is not possible to reproduce the excess at the late epochs with a simple (Arnett 1982) model including $0.42 M_{\odot}$ of ${ }^{56} \mathrm{Ni}$ and a large ejecta mass of $10.4 M_{\odot}$ (red line in Fig. 8). Here, following T16 and the measurements of the Fe II line P Cygni absorption velocity, we assumed the ejecta to expand with a bulk velocity of $6000 \mathrm{~km} \mathrm{~s}^{-1}$ and to have uniform density. These assumptions imply a kinetic energy of $2.2 \times 10^{51} \mathrm{erg}$ for the radioactive model.

An additional source of power is needed to fit the early and the late-time light curves simultaneously. We test if a magnetar can fit both phases (blue line in Fig. 8). Indeed, using the models presented in Inserra et al. (2013) and derived from Kasen \& Bildsten (2010), we obtain a good fit with an ejecta mass of $1.2 M_{\odot}$, magnetic field intensity $\mathrm{B}=2.6 \times 10^{14} \mathrm{G}$, and an initial spin period of $14.8 \mathrm{~ms}$. However, the small ejecta mass required to fit the late epochs does not perfectly reproduce the early peak, which is rather broad. This model assumes again an ejecta bulk velocity of $6000 \mathrm{~km} \mathrm{~s}^{-1}$ and uniform density, implying a low kinetic energy of $0.2 \times 10^{51} \mathrm{erg}$ ).
The model with the magnetar alone also produces an earlier peak than what is observed (see inset of Fig. 8). Furthermore, we know that iPTF15dtg is somewhat similar to other SE SNe. It is not super-luminous and displays a perfectly normal spectral evolution. It is therefore reasonable to assume that it ejected some amount of ${ }^{56} \mathrm{Ni}$, like other SE SNe. This ${ }^{56} \mathrm{Ni}$ will not strongly influence the late-time light curve, where another source, like a magnetar, will dominate. Therefore, we try a hybrid model where the input powering is both a magnetar and radioactive ${ }^{56} \mathrm{Ni}$ (green lines in Fig. 8). In this case, we obtain a better fit at earlier epochs (a longer rise time, see solid green light in the inset of Fig. 8) where the ${ }^{56} \mathrm{Ni}$ dominates, and a good fit at late epochs where a magnetar is prominent. The best-fit ejecta mass turns out to be about $3.5 M_{\odot}$, the ${ }^{56} \mathrm{Ni}$ mass is $0.29 M_{\odot}$, the spin period $19.1 \mathrm{~ms}$, and the magnetic field $\mathrm{B}=1.6 \times 10^{14} \mathrm{G}$. Given the same assumptions on the ejecta velocity as in the other models, the kinetic energy turns out to be $0.8 \times 10^{51} \mathrm{erg}$. These are physically reasonable values, which describe iPTF15dtg as a normal SE SN where a magnetar modifies the peak and dominates the emission at late epochs, making this SN extraordinarily bright more than two years after explosion.

The modeling presented in this section does not include any possible gamma-ray and spin-down energy leakage, as presented in Wang et al. (2015). This could play an important role in the late-time light curve. We test this by running a few models (both magnetar and magnetar+radioactivity) allowing for such energy 


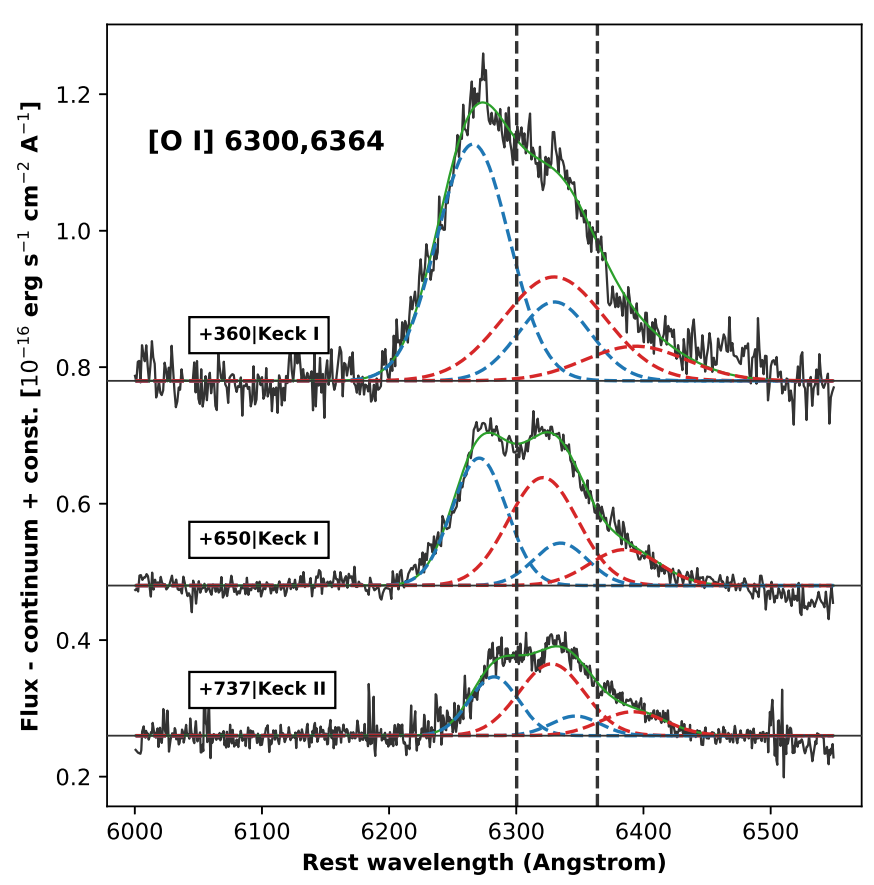

Fig. 6. Continuum-subtracted [O I] $\lambda 6300,6364$ line profile fit. Two Gaussians (a blueshifted and a redshifted component) for each of the two lines ( 6300 and $6364 \AA$ ) were used. The normalization of the two lines was fixed so that the $6300 \AA$ line has three times the flux of the $6364 \AA$ line. The centroids of the corresponding components of the two lines were also fixed to be $64 \AA$ apart.

leakage. We consider gamma-ray opacities between 0.01 and 0.2 $(0.01,0.03,0.1,0.2) \mathrm{cm}^{2} \mathrm{~g}^{-1}$, as suggested in Wang et al. (2015). The hybrid model provides a good fit for $\kappa_{\gamma}=0.2 \mathrm{~cm}^{2} \mathrm{~g}^{-1}$, and a moderately good fit for $\kappa_{\gamma}=0.1 \mathrm{~cm}^{2} \mathrm{~g}^{-1}$. For lower gammaray opacities it does not provide a good fit. The simple magnetar model provides a moderately good fit for $\kappa_{\gamma}=0.1 \mathrm{~cm}^{2} \mathrm{~g}^{-1}$ and $\kappa_{\gamma}=0.03 \mathrm{~cm}^{2} \mathrm{~g}^{-1}$. All these fits are however poorer than those obtained assuming full trapping. If we consider the best model among those including energy leakage, that is, the hybrid model with $\kappa_{\gamma}=0.2 \mathrm{~cm}^{2} \mathrm{~g}^{-1}$, this provides an ejecta mass of $3.4 M_{\odot}$, similar to the case of the full-trapping hybrid model, but with a much lower (more than two orders of magnitude) magnetic-field intensity and spin period (one order of magnitude). At epochs later than $\sim 700 \mathrm{~d}$ the bolometric light curve starts to decline faster as compared to the previous $\sim 500 \mathrm{~d}$, and the hybrid model that allows for the energy leakage fits this phase better than the models that do not allow for the leakage. If we consider the best magnetar model $\left(\kappa_{\gamma}=0.1 \mathrm{~cm}^{2} \mathrm{~g}^{-1}\right)$, again we find a low ejecta mass $\left(0.5 M_{\odot}\right)$, relatively similar to the best magnetar model with full trapping, but a much lower $B$ and spin period (two and one order of magnitude, respectively). We note that the required gamma-ray opacity $\kappa_{\gamma}$ to get a good late-phase magnetar fit $\left(0.2 / 0.1 \mathrm{~cm}^{2} \mathrm{~g}^{-1}\right)$ is relatively high compared with those estimated for the SLSN magnetar fits.

Also, T16 examined the possibility that the very early lightcurve excess shown by iPTF15dtg could arise from a magnetarpowered shock breakout (Kasen et al. 2016). However, for the parameters derived from both the magnetar model and the hybrid model, the maximum luminosity of this first peak would be one order of magnitude larger than what is observed, and the same is true for the timescale of the breakout. This is true also for the models where we do not assume full trapping.

\section{Discussion}

\subsection{Powering mechanism}

To conclude on the powering mechanism of iPTF15dtg, this cannot be only due to the regular radioactive decay powering as in other $\mathrm{SE} \mathrm{SNe}$, given its extraordinary slow late-time decline. If the bolometric light curve between 300 and $400 \mathrm{~d}$ were entirely powered by radioactivity, a ${ }^{56} \mathrm{Ni}$ mass of $0.8 M_{\odot}$ would be required. However, this amount of radioactive material would also imply a peak bolometric light curve of $10^{43} \mathrm{erg} \mathrm{s}^{-1}$, which is a factor of two brighter than what we observe. We also note that other isotopes such as ${ }^{57} \mathrm{Co}$ and ${ }^{44} \mathrm{Ti}$ do not contribute significantly to the light curve until epochs later than our observations (see Fransson \& Kozma 1993, and the case of SN 1987A), so they cannot account for the excess of iPTF15dtg.

A magnetar alone might power the light curve of iPTF15dtg. Our magnetar model fits reasonably well the evolution across the entire 800-d period during which we observed iPTF15dtg. However, the fit at early epochs is not perfect, as the peak epoch of iPTF15dtg occurs later than that of our magnetar model. Furthermore, we must consider that SN iPTF15dtg in many respects is similar to a normal SE SNe, and thus we expect to have some radioactive material also contributing to the luminosity. Therefore, a hybrid model with radioactive material dominating at early epochs and a magnetar dominating the nebular phase is our favorite scenario and gives the best fit to the bolometric light curve. We note that when we consider the possibility of spin-down energy leakage in our models instead of full trapping, we generally obtain lower-quality fits, but we can however reproduce the light curve obtaining similar ejecta masses to the models that assume full trapping. However, these models are characterized by a much lower magnetic field intensity and spin period.

Based on the spectral and light-curve comparison to the interacting SE SN 2010mb, we consider it less likely that circumstellar interaction plays a major role as a source of luminosity for iPTF15dtg, even though we cannot completely exclude its presence. We argue that the spectra of iPTF15dtg are too similar to those of other regular SE SNe to hide a CSM interaction responsible for an excess in luminosity of several magnitudes. However there are a few arguments that might be in favor of the CSM-interaction mechanism. First, both magnetar and radioactive powering are considered as smooth processes that produce a smoothly declining luminosity. However, the light curve of iPTF15dtg shows some variability and a late-time break that might be more naturally explained by CSM interaction. However, it is not excluded that a magnetar-powered SN can produce fluctuations in the light curve, as observed in some SLSNe-I (e.g., Nicholl et al. 2016b). We also noted that the spectra of iPTF15dtg at late times have a blue excess that is slightly more prominent than in other SE SNe, even though it is not as pronounced as in SN 2010mb. This excess looks quite similar to the late emission from SNe IIn (except for the $\mathrm{H}$ lines), where the blue excess is dominated by Fe II emission from CSM interaction. However, the light-curve excess is too strong to be explained by this blue excess. The late-time luminosity may also be explained in terms of CSM interaction, adopting for example a model from Chatzopoulos et al. (2013) with the collision between the ejecta and a CSM shell of $0.1 M_{\odot}$ occurring $130 \mathrm{~d}$ post-explosion. However, such a scenario is difficult to reconcile with the spectral observations as previously explained.

Given the long-lasting emission of iPTF15dtg we might also consider more exotic powering mechanisms, such as fallbackaccretion powering. In the case of iPTF14hls, an extraordinary 


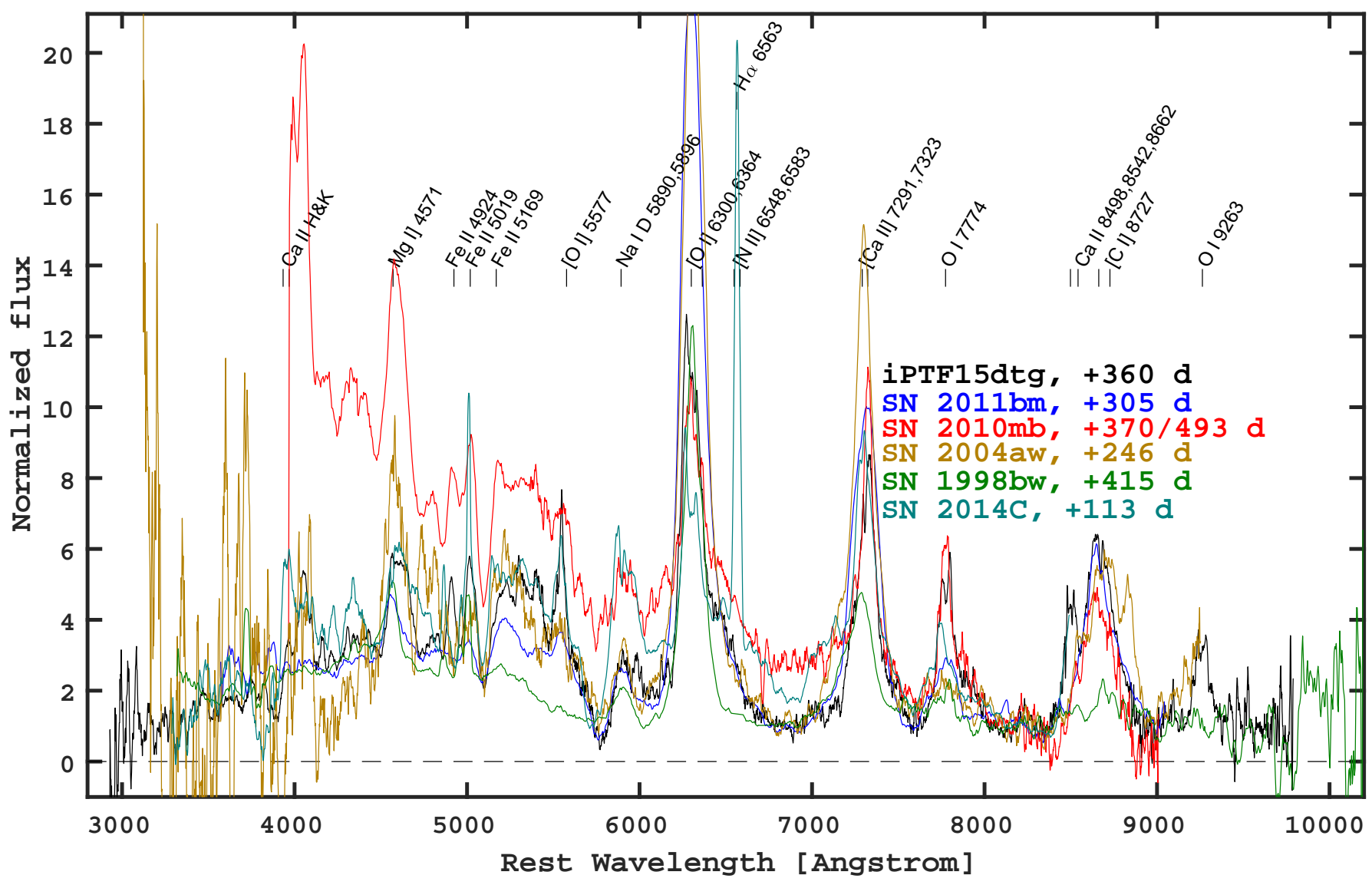

Fig. 7. Normalized (divided by the median flux between 8000 and $8400 \AA$ A) spectra of iPTF15dtg (black), SN 2011bm (blue), SN 2010mb (red), SN 1998bw (green), SN 2004aw (orange), and SN 2014C (blue-green) at nebular epochs. All spectra were corrected for extinction. The main features are marked as in Fig. 5. The phases are reported for each SN. Supernova $2010 \mathrm{mb}$ is clearly different as its continuum is remarkably bluer than for the other SNe. Supernova 2014C shows strong $\mathrm{H} \alpha$ emission.

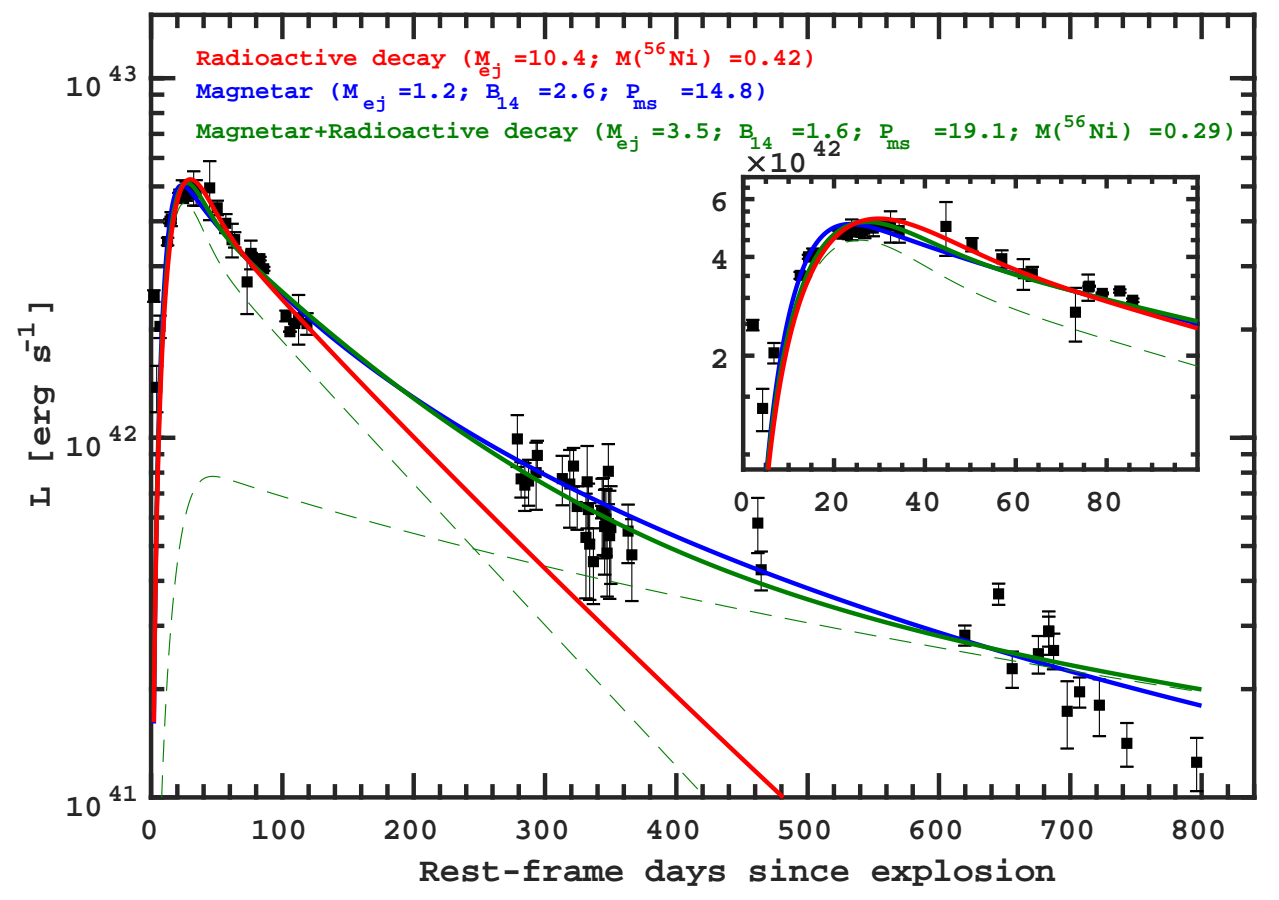

Fig. 8. Bolometric light curve of iPTF15dtg from explosion until $800 \mathrm{~d}$ (rest-frame). For the first $150 \mathrm{~d}$ we adopt the bolometric light curve that was computed in T16. After that, we make use of our spectra to compute late-time bolometric corrections to the $r$-band light curve and hence obtain the luminosity at late epochs. We fit the bolometric light curve with three models: a purely radioactive model (red), a magnetar model (blue) and a hybrid model (green). The best fit parameters are reported in the corresponding colors. The two components of the hybrid model are shown by green dashed lines (at early epochs the radioactivity dominates, at late epochs the magnetar is prominent). The inset shows a zoomed view of the fits around the light curve peak. spectroscopically normal SN II with a very long-lasting emission, the late-time bolometric light curve was compared to a power law with index $-5 / 3$ (Sollerman et al. 2019), which is expected for a fallback-accretion-powering scenario. If we compare the light curve of iPTF15dtg with this expected trend, we find that the emission of iPTF15dtg at epochs later than $250 \mathrm{~d}$ are 
overall in agreement with such a power law. However, we notice that in the late-time spectra of iPTF15dtg we detect strong [O I] emission. Oxygen, which sits in the inner layers of the ejecta, should be among the first elements to fall back into the central black hole, and therefore should not leave a strong signature in the spectra. Another possibility is that the late-time emission of iPTF15dtg is powered by a light echo (see e.g., Chevalier 1986); however without near-infrared observations it is hard to assess this scenario.

\subsection{Progenitor implications}

T16 observed iPTF15dtg up to around $150 \mathrm{~d}$, and favor a radioactive model for this $\mathrm{SN}$, with a large ejecta mass of $10.3 M_{\odot}$ and a ${ }^{56} \mathrm{Ni}$ mass of $0.42 M_{\odot}$. This implies a massive, possibly single progenitor star of at least $35 M_{\odot}$, something very unusual among the SE SNe. However, here we show that the late-time bolometric light curve of iPTF15dtg displays - for the first time for a normal SE SN - a decline rate much slower than that of ${ }^{56} \mathrm{Co}$, implying a different or additional mechanism powering the luminosity. Our hybrid model, where the contribution of a magnetar is also included and which provides the best fit to the data, implies a lower ejecta mass for iPTF15dtg of about $3.6 M_{\odot}$. This is in line with what has been presented in the literature for regular SE SNe (see e.g., Taddia et al. 2015, 2018; Lyman et al. 2016) and might imply a lower initial mass for the progenitor star of iPTF15dtg, and therefore a likely origin in a binary system given that this $\mathrm{SN}$ was stripped of its $\mathrm{H}$ and $\mathrm{He}$ envelopes.

There is a caveat to this result. If the magnetar spin-down energy is not fully trapped, which we are assuming in our models, then a larger ejecta mass would be required to sustain such a slow luminosity decline, and a (single) massive star progenitor scenario for SN iPTF15dtg might still be allowed. However, if we include in our model the leakage of hard emission as presented by Wang et al. (2015) for SLSNe powered by magnetars, we would not be able to fit both the peak and tail of the bolometric light curve of iPTF15dtg simultaneously. The reason is that the leakage would push the late-time luminosity of our model towards lower values than those observed. Even for a large ejecta mass of $10 M_{\odot}$, the luminosity at $800 \mathrm{~d}$ would be reduced by a factor of four, preventing a good fit of the light-curve tail. However, it is not clear that the leakage of energy from a magnetar occurs as leakage of hard emission, as assumed by Wang et al. (2015). In fact the assumption of full energy trapping might not apply after $700 \mathrm{~d}$, where the light curve begins to drop faster.

\section{Conclusion}

We observed supernova iPTF15dtg until $\sim 840$ d post-explosion. The spectra are similar to those of regular SE SNe. On the contrary, the late-time light curve declines very slowly compared to ${ }^{56}$ Co decay, which is unprecedented among regular SE $\mathrm{SNe}$. The bolometric light curve is well reproduced by a model including both radioactivity (dominating at early epochs) and a magnetar (powering the late emission). This model allows us to revise the ejecta mass of iPTF15dtg to a lower value compared to what was discussed in T16, implying a lower initial mass and thus a possible binary scenario for the progenitor star of iPTF15dtg.

Acknowledgements. FT and JS gratefully acknowledge support from the Knut and Alice Wallenberg Foundation. JS acknowledges the support of Vetenskapsrådet through VR grants 2012-2265 and 2017-03699. This work is partly based on observations made with the Nordic Optical Telescope, operated by the Nordic Optical Telescope Scientific Association at the Observatorio del Roque de los Muchachos, La Palma, Spain, of the Instituto de Astrofisica de Canarias. The data presented here were obtained in part with ALFOSC, which is provided by the Instituto de Astrofisica de Andalucia (IAA) under a joint agreement with the University of Copenhagen and NOTSA. This work is partly based on observations made with DOLoRes@TNG. Based on observations obtained with the Samuel Oschin Telescope 48-inch and the 60-inch Telescope at the Palomar Observatory as part of the intermediate Palomar Transient Factory (iPTF) project, a scientific collaboration among the California Institute of Technology, Los Alamos National Laboratory, the University of Wisconsin, Milwaukee, the Oskar Klein Center, the Weizmann Institute of Science, the TANGO Program of the University System of Taiwan, and the Kavli Institute for the Physics and Mathematics of the Universe.

\section{References}

Arnett, W. D. 1982, ApJ, 253, 785

Ben-Ami, S., Gal-Yam, A., Mazzali, P. A., et al. 2014, ApJ, 785, 37 Cenko, S. B., Fox, D. B., Moon, D.-S., et al. 2006, PASP, 118, 1396 Chatzopoulos, E., Wheeler, J. C., Vinko, J., Horvath, Z. L., \& Nagy, A. 2013, ApJ, 773, 76

Chevalier, R. A. 1986, ApJ, 308, 225

Fransson, C., \& Kozma, C. 1993, ApJ, 408, L25

Fremling, C., Sollerman, J., Taddia, F., et al. 2016, A\&A, 593, A68 Inserra, C., Smartt, S. J., Jerkstrand, A., et al. 2013, ApJ, 770, 128 Kasen, D., \& Bildsten, L. 2010, ApJ, 717, 245

Kasen, D., Metzger, B. D., \& Bildsten, L. 2016, ApJ, 821, 36 Jerkstrand, A., Ergon, M., Smartt, S. J., et al. 2015, A\&A, 573, A12 Lyman, J. D., Bersier, D., \& James, P. A. 2014, MNRAS, 437, 3848 Lyman, J. D., Bersier, D., James, P. A., et al. 2016, MNRAS, 457, 328 Margutti, R., Kamble, A., Milisavljevic, D., et al. 2017, ApJ, 835, 140 Masci, F. J., Laher, R. R., Rebbapragada, U. D., et al. 2017, PASP, 129, 014002 Milisavljevic, D., Margutti, R., Kamble, A., et al. 2015, ApJ, 815, 120 Milisavljevic, D., Patnaude, D. J., Chevalier, R. A., et al. 2018, ApJ, 864, L36 Nicholl, M., Berger, E., Margutti, R., et al. 2016a, ApJ, 828, L18 Nicholl, M., Berger, E., Smartt, S. J., et al. 2016b, ApJ, 826, 39 Oke, J. B., Cohen, J. G., Carr, M., et al. 1995, PASP, 107, 375 Patat, F., Cappellaro, E., Danziger, J., et al. 2001, ApJ, 555, 900 Rahmer, G., Smith, R., \& Velur, V. 2008, Proc. SPIE, 7014 Sollerman, J., Taddia, F., Arcavi, I., et al. 2019, A\&A, 621, A30 Stritzinger, M., Taddia, F., Fransson, C., et al. 2012, ApJ, 756, 173 Taddia, F., Sollerman, J., Leloudas, G., et al. 2015, A\&A, 574, A60 Taddia, F., Fremling, C., Sollerman, J., et al. 2016, A\&A, 592, A89 Taddia, F., Stritzinger, M. D., Bersten, M., et al. 2018, A\&A, 609, A136 Taubenberger, S., Pastorello, A., Mazzali, P. A., et al. 2006, MNRAS, 371, 1459

Taubenberger, S., Valenti, S., Benetti, S., et al. 2009, MNRAS, 397, 677 Valenti, S., Taubenberger, S., Pastorello, A., et al. 2012, ApJ, 749, L28 Wang, S. Q., Wang, L. J., Dai, Z. G., \& Wu, X. F. 2015, ApJ, 799, 107 Wheeler, J. C., Johnson, V., \& Clocchiatti, A. 2015, MNRAS, 450, 1295 Yaron, O., \& Gal-Yam, A. 2012, PASP, 124, 668 